OPEN ACCESS

Edited by:

Akio Adachi,

Tokushima University, Japan

Reviewed by:

Xiaoming Sun

Ragon Institute of MGH, MIT and

Harvard, United States

Florian Krammer,

Icahn School of Medicine at Mount

Sinai, United States

*Correspondence:

Xinan Jiao

jiao@yzu.edu.cn

Xiufan Liu

xfliu@yzu.edu.cn

Specialty section:

This article was submitted to

Virology,

a section of the journa

Frontiers in Microbiology

Received: 01 July 2017 Accepted: 15 September 2017 Published: 26 September 2017

Citation:

Hu Z, Jiao X and Liu X (2017) Antibody Immunity Induced by H7N9 Avian Influenza Vaccines: Evaluation

Criteria, Affecting Factors, and Implications for Rational Vaccine Design. Front. Microbiol. 8:1898. doi: 10.3389/fmicb.2017.01898

\section{Antibody Immunity Induced by H7N9 Avian Influenza Vaccines: Evaluation Criteria, Affecting Factors, and Implications for Rational Vaccine Design}

\author{
Zenglei Hu ${ }^{1,2}$, Xinan Jiao ${ }^{1,2,3,4 *}$ and Xiufan Liu 1, 2,3,4* \\ ${ }^{1}$ Animal Infectious Disease Laboratory, School of Veterinary Medicine, Yangzhou University, Yangzhou, China, ${ }^{2}$ Jiangsu \\ Co-innovation Center for Prevention and Control of Important Animal Infectious Diseases and Zoonoses, Yangzhou \\ University, Yangzhou, China, ${ }^{3}$ Jiangsu Key Laboratory of Zoonosis, Yangzhou University, Yangzhou, China, ${ }^{4}$ Key Laboratory \\ of Prevention and Control of Biological Hazard Factors (Animal Origin) for Agri-Food Safety and Quality, Ministry of Agriculture \\ of China, Yangzhou University, Yangzhou, China
}

Severe H7N9 avian influenza virus (AIV) infections in humans have public health authorities around the world on high alert for the potential development of a human influenza pandemic. Currently, the newly-emerged highly pathogenic avian influenza A (H7N9) virus poses a dual challenge for public health and poultry industry. Numerous H7N9 vaccine candidates have been generated using various platforms. Immunization trials in animals and humans showed that H7N9 vaccines are apparently poorly immunogenic because they induced low hemagglutination inhibition and virus neutralizing antibody titers. However, H7N9 vaccines elicit comparable levels of total hemagglutinin ( $\mathrm{HA}$ )-reactive lgG antibody as the seasonal influenza vaccines, suggesting $\mathrm{H} 7 \mathrm{~N} 9$ vaccines are as immunogenic as their seasonal counterparts. A large fraction of overall IgG antibody is non-neutralizing antibody and they target unrecognized epitopes outside of the traditional antigenic sites in HA. Further, the Treg epitope identified in $\mathrm{H} 7$ HA may at least partially contribute to regulation of antibody immunity. Here, we review the latest advances for the development of $\mathrm{H} 7 \mathrm{~N} 9$ vaccines and discuss the influence of serological criteria on evaluation of immunogenicity of H7N9 vaccines. Next, we discuss factors affecting antibody immunity induced by H7N9 vaccines, including the change in antigenic epitopes in HA and the presence of the Treg epitope. Last, we present our perspectives for the unique features of antibody immunity of H7N9 vaccines and propose some future directions to improve or modify antibody response induced by H7N9 vaccines. This perspective would provide critical implications for rational design of $\mathrm{H} 7 \mathrm{~N} 9$ vaccines for human and veterinary use.

Keywords: antibody immunity, H7N9 vaccines, antigenic epitope, Treg, vaccine design

\section{INTRODUCTION}

Avian influenza A (H7N9) virus emerged in China in 2013, causing severe human infections with high mortality (Gao et al., 2013). Up to now, mainland China has experienced five epidemic waves of H7N9 influenza. As of 25 July, 2017, a total of 1,557 laboratory-confirmed cases of human infection with H7N9 avian influenza virus (AIV), including at least 605 deaths, have been 
reported to WHO (http://101.96.10.63/www.who.int/entity/ influenza/human_animal_interface/Influenza_Summary_IRA_ HA_interface_07_25_2017.pdf?ua\$=\$1). Of note, the fifth epidemic of human cases of H7N9 infection occurred earlier, spread to more areas and infected more people than in any earlier waves (Iuliano et al., 2017; Wang X. et al., 2017). H7N9 AIVs from the first four waves are low pathogenic in chickens and most viruses were isolated from healthy birds in live poultry market (LPM). However, on Feb 19, 2017, H7N9 virus with a four basic amino acid insertion in the cleavage site of the hemagglutinin (HA) was detected in two patients with H7N9 virus infection in Guangdong province (http://www.who.int/csr/ don/27-february-2017-ah7n9-china/en/). Such strains fulfill the requirements for the classification as highly pathogenic avian influenza virus (HPAIV). Subsequently, more HPAIV H7N9 strains were isolated from humans, poultry and environment samples from LPM (Yang and Liu, 2017; Zhang et al., 2017) (http://www.oie.int/wahis_2/public\%5C..\%5Ctemp\%5Creports/ en_fup_0000024044_20170614_142756.pdf). Although, humanto-human transmission of H7N9 AIVs has been limited to a few of family clusters, the presence of multiple mammalian-adaption molecular markers has raised concerns about the potential of H7N9 viruses to be transmissible among humans and cause a next pandemic (Li et al., 2017).

In addition, according to the reports from World Animal Health Organization, H7N9 HPAIV has already caused nine severe outbreaks in poultry flocks in China since March, 2017, resulting in over 800,000 chickens being destroyed (http:// www.oie.int/wahis_2/public\%5C..\%5Ctemp\%5Creports/en_ fup_0000024044_20170614_142756.pdf). Therefore, currently, H7N9 AIV poses a dual challenge for public health and poultry industry.

Vaccination is the most effective intervention to prevent influenza. However, no vaccines for H7N9 influenza are available for human and veterinary use. Since the first emergence of H7N9 influenza in 2013, a large number of vaccine candidates have been generated using various vaccine platforms. Most of them have been assessed in animal trials, but only a few have been examined in clinical trials in volunteers. The traditional criteria for immunogenicity evaluation are based on the classical serological tests, i.e., hemagglutination inhibition (HI) and virus neutralization (VN) assays. Immunogenicity of $\mathrm{H} 7 \mathrm{~N} 9$ vaccines are considered to be poor based on low $\mathrm{HI}$ and $\mathrm{VN}$ titers they induced. However, the latest findings suggest that an $\mathrm{H} 7 \mathrm{~N}$ 9 vaccine is as immunogenic as $\mathrm{H} 1 \mathrm{~N} 1$ and $\mathrm{H} 3 \mathrm{~N} 2$ influenza vaccines in mice because they induced comparable total IgG antibody titers as measured by ELISA. Moreover, antibody immunity of H7N9 vaccines is also affected by multiple factors, e.g., the variation of the antigenic sites and the presence of putative inhibitory Treg epitope in HA. Here, we summarize the current advances in the development of H7N9 vaccines. Then we discuss the change of criteria for evaluation of $\mathrm{H} 7 \mathrm{~N} 9$ vaccine immunogenicity and discuss the factors affecting antibody immunity induced by H7N9 vaccines. Finally, we present our perspectives and propose directions for rational vaccine design or modification.

\section{H7N9 INFLUENZA VACCINES IN ANIMAL TRIALS}

Numerous H7N9 vaccine candidates have been generated using various technologies, including the traditional approaches, such as whole virus inactivation, split-virion and live attenuated vaccine, and cutting-edge technologies, such as virus-like particles (VLP), recombinant protein expression, nucleic acid vaccines and viral vector vaccines. Their immunogenicity and efficacy were assessed in different animal models.

A new and attractive vaccine platform based on synthetic selfamplifying mRNA allows generation of any influenza vaccine within 8 days once the sequence is available. The HA mRNA of H7N9 strain A/Shanghai/2/2013 encapsulated with lipid nanoparticle was immunogenic in mice, inducing high $\mathrm{HI}$ and VN antibody titers (Hekele et al., 2013). In addition, mRNA vaccines against $\mathrm{H} 10 \mathrm{~N} 8$ and H7N9 influenza generated a rapid and robust immune response in mice, ferrets and non-human primates (Bahl et al., 2017). However, no H7N9 mRNA vaccine candidate has been evaluated in clinical trials, and thus the lack of safety and efficacy data in humans may restrain the potential of mRNA vaccines to be used for influenza pandemic.

Another promising platform allowing rapid production of H7N9 vaccine is the VLP technology. Influenza VLPs are formed by a self-assembly process incorporating the surface proteins, the HA and neuraminidase (NA), into the matrix (M1) protein. The size and morphology of VLPs resemble those of influenza virus particles. The major advantage of VLPs is that they are noninfectious due to the absence of viral nucleic acid in the process of VLP assembly. Two vaccinations with the first H7N9 VLP vaccine induced high HI antibody titers and provided a good protection against H7N9 challenge in mice (Smith et al., 2013). Inclusion of adjuvant enhanced immunogenicity and efficacy of this VLP candidate. A similar VLP formulated with two saponin-based adjuvants was further tested in ferrets. Two doses of non-adjuvanted VLP in ferrets elicited high $\mathrm{HI}$ and VN titers and addition of either adjuvant significantly increased immunogenicity and protection against H7N9 challenge (Liu Y. V. et al., 2015). Additionally, a VLPlike H7N9 vaccine generated by baculovirus surface-displaying approach was immunogenic and completely protected mice from H7N9 challenge (Prabakaran et al., 2014). Multi-HA VLPs have been also generated to provide protection against different subtypes of H2, H7, and H9 simultaneously (Pushko et al., 2011). Generally, most H7N9 VLP vaccines are immunogenic in animal models and the adjuvant is usually added to provide better protection against virus replication and shedding.

Recombinant protein technology is also a promising approach for timely preparation of H7N9 vaccines without handling live viruses. Flublok seasonal influenza vaccines produced in baculovirus expression system do not contain adjuvants and thus a high dose ( $45 \mu \mathrm{g}$ of HA) is needed to induce a robust antibody immunity (Cox et al., 2008). A comparative study of immunogenicity of HA antigens from different subtypes revealed that the HA proteins of H7N7 and H7N2 induced significantly lower neutralizing antibody titers in mice than did HAs of the 
seasonal influenza viruses (Blanchfield et al., 2014). For H7N9 HA-based vaccines, the full-length HA, HA2 subunit and HA globular head were expressed in either insect cells or E. coli (Pushko et al., 2015; Song et al., 2015, 2016; Cao et al., 2016b). The HA protein from a human-origin H7N9 strain expressed in insect cells formed subviral particles (SVP), while two intranasal immunization of the SVP induced minimal HI titers in mice without MPL adjuvant. The addition of MPL adjuvant dramatically increased $\mathrm{HI}$ antibody titers. Interestingly, nonadjuvanted SVP vaccine still provided $80 \%$ protection against H7N9 challenge (Pushko et al., 2015). Similarly, the HA globular head (HA1) also induced low HI titers even after two or three shots (Song et al., 2015, 2016). However, the HA2 subunit was immunogenic in mice without adjuvant and a single dose with adjuvant increased anti-HA2 IgG titers and protected mice from H7N9 challenge (To et al., 2015). Usage of different types of adjuvants can enhance antibody immunity. It is apparent that the HA-based subunit vaccines are poorly immunogenic based on low $\mathrm{HI}$ or $\mathrm{VN}$ titers.

The traditional approaches for engineering of inactivated $\mathrm{H} 7 \mathrm{~N} 9$ vaccines require handling of infectious live viruses grown in eggs or cell cultures followed by inactivation or virion disruption. The common practice is to generate reassortant viruses containing the HA and NA genes from the seasonal or pandemic influenza viruses and the remaining six internal genes from an highly egg-adapted strain $\mathrm{A} / \mathrm{PR} / 8 / 34$ using reverse genetics. Numerous such reassortant viruses carrying the HA and NA genes from H7N9 strains have been generated. In general, non-adjuvanted whole-virus inactivated vaccines (WVIVs) induced antibody titers in a dose-dependent manner in mice, ferrets, guinea pigs, and macaques. High levels of HI, VN and IgG antibody and good protections from H7N9 challenge were only observed for high doses (Chu et al., 2014; Pan et al., 2014; Wong et al., 2014; Wodal et al., 2015; Seo and Kim, 2016). H7N9 split-virion and WVIVs showed distinctive patterns of immunogenicity in different animal models (Duan et al., 2014; Wong et al., 2014; Wu et al., 2014; Ou et al., 2016). A recent study showed that an inactivated $\mathrm{H} 7 \mathrm{~N} 9$ vaccine containing a replaced H3 HA transmembrane domain (TM) induced significantly higher $\mathrm{HI}$ titers, HA-specific IgG titers and IFN- $\gamma$ production and provided improved interclade protection in mice compared to the vaccine containing the wild-type $\mathrm{H} 7 \mathrm{HA}$ (Wang Y. et al., 2017).

Live attenuated influenza vaccines (LAIV) have some advantages over WVIVs. LAIVs can establish mucosal immunity in the respiratory tract and induce cellular immunity. LAIVs based on both Leningrad/134/17/57 and A/Ann Arbor/6/60 master donor viruses were highly immunogenic in ferrets, mice and guinea pigs and are efficacious against lethal H7N9 challenge (Chen et al., 2014; Kong et al., 2015; Yang et al., 2015; de Jonge et al., 2016; Isakova-Sivak et al., 2017). However, the safety of H7N9 LAIVs must be evaluated sufficiently because the two surface proteins are derived from fatal H7N9 isolates. Moreover, viral-vectored $\mathrm{H} 7 \mathrm{~N} 9$ vaccines based on modified vaccinia virus, vesicular stomatitis virus, and human adenovirus were also immunogenic and efficacious in animal models (Kreijtz et al., 2015; Cao et al., 2016a; Wongthida et al., 2016). However, an
H7HA-expressing vaccine based on parainfluenza virus 5 can provide full protection against $\mathrm{H} 7 \mathrm{~N} 9$ infection in mice, whereas it failed to induce any measurable antibody titers, suggesting a potential role of cellular immunity in protection (Chia et al., 2015).

In the veterinary field, poultry trials also present supporting evidence. For poultry influenza vaccines, HI antibody is usually used as a serological surrogate of protection and an $\mathrm{HI}$ titer above 16 is thought to be clinically protective. A Newcastle disease virus (NDV)-vectored H7 HA vaccine induced low H7specific HI titers (13 after booster), whereas this vaccine provided 90\% protection against HPAIV H7N7 challenge (Park et al., 2006). Similarly, a NDV-based vaccine expressing HA of H7N9 A/Anhui/1/2013 strain was low immunogenic in chickens, with only $20 \%$ of chickens being HI-positive and HI titers were around 10 after the first immunization. In contrast, in the same study, a NDV-based $\mathrm{H} 5$ vaccine induced seroconversion in $50 \%$ of chickens and $\mathrm{HI}$ titers were around 20 after the first vaccination (Liu Q. et al., 2015). In addition, our team has generated H7 HAexpressing vaccines based on different vectors, including NDV, herpesvirus of turkeys and chimeric avian paramyxovirus 2 , and evaluated their immunogenicity in chickens. All these vaccine candidates consistently induced undetectable or very low $\mathrm{HI}$ antibody titers even after booster immunization (unpublished data).

\section{H7N9 INFLUENZA VACCINES IN CLINICAL TRIALS}

Although, diverse H7N9 vaccine candidates were generated and positive results were obtained from animal trials, only a few have been investigated in clinical trials in volunteers. Only data from clinical trials for VLP, subunit, split-virion, and WVIVs were listed and discussed here (Table 1). For human influenza vaccines, an $\mathrm{HI}$ antibody titer above 40 is recognized as an immunological correlate corresponding to a $50 \%$ reduction in the risk of influenza virus infection. Novavax's H7N9 VLP vaccine based on an avian-origin virus had low immunogenicity in humans without adjuvant. Only 6 and 16\% seroconversion were observed for 15 and $45 \mu \mathrm{g}$ vaccine dose, respectively, and $\mathrm{HI}$ titers were below 10 after two doses. The addition of ISCOMATRIX adjuvant significantly increased antibody titers after the second dose (Fries et al., 2013). Similarly, subunit vaccines were also poorly immunogenic in adults, with 3 and $7 \%$ seroconversion rate as determined by $\mathrm{HI}$ and $\mathrm{VN}$ assays. The $\mathrm{HI}$ and $\mathrm{VN}$ titers were about 5 , with no increase after the second dose. The inclusion of MF59 adjuvant dramatically improved immunogenicity of the vaccine (Bart et al., 2014). A clinical trial of split-virion vaccines formulated with or without MF59 adjuvant gave similar results that the non-adjuvanted vaccines were low immunogenic, whereas the MF59-adjuvanted immunogens induced a robust antibody immune response (Mulligan et al., 2014). Other clinical trials also demonstrated that all tested H7N9 vaccines in different formulations in the absence of adjuvant had poor immunogenicity in human beings (Jackson et al., 2015; Madan et al., 2016). Furthermore, clinical trials of 


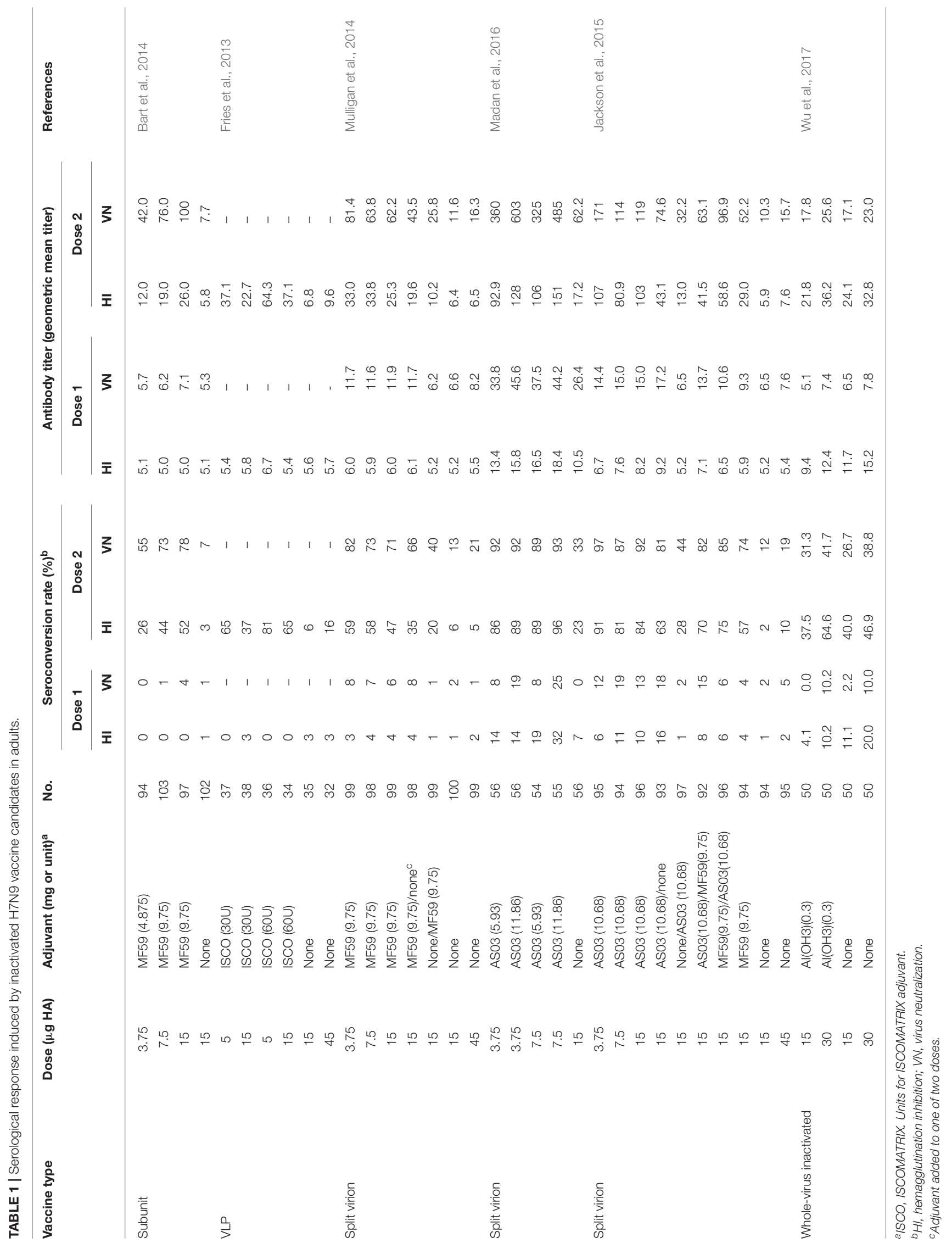


vaccines for $\mathrm{H} 7 \mathrm{~N} 1$ and $\mathrm{H} 7 \mathrm{~N} 7$ subtypes also showed that they were poorly immunogenic in humans (Cox et al., 2009; Couch et al., 2012). In contrast, the monovalent $\mathrm{pH} 1 \mathrm{~N} 1$ vaccine and trivalent seasonal vaccine were highly immunogenic in healthy adult subjects without adjuvant, with seroconversion rate above 80\% (Griffin et al., 2011; Grohskopf et al., 2013). However, a clinical trial of an H7N9 WVIV in Taiwan population showed that a dose of $15 \mu \mathrm{g}$ HA induced a seroconversion rate of $40 \%$ and high $\mathrm{HI}$ titers without adjuvant. A higher dose ( $30 \mu \mathrm{g} \mathrm{HA})$ or the inclusion of $\mathrm{AI}(\mathrm{OH} 3)$ adjuvant improved antibody response (Wu et al., 2017). It is obvious that induction of low HI titers is a common character for $\mathrm{H} 7$ subtype influenza vaccines. The addition of adjuvant did increase immunogenicity of the vaccines in humans, whereas the adjuvant is not currently used in standard seasonal influenza vaccines. Moreover, experts have raised some concerns about the use of adjuvants in influenza vaccination because of the likely association between adjuvanted influenza vaccination campaign and narcolepsy in children and young people in Europe (Nohynek et al., 2012; Partinen et al., 2012; Duan et al., 2014).

\section{CRITERIA FOR EVALUATION OF IMMUNOGENICITY OF H7N9 VACCINE CANDIDATES}

Although, $\mathrm{HI}$ and VN antibodies are well understood to correlate with protection for the seasonal influenza vaccines, it is not clear whether these serological assays also correlate with protection against avian influenza viruses such as $\mathrm{H} 7$ subtype. It is vital to point out that evaluation of immunogenicity of most H7N9 vaccines in animal models and humans described above was based on the traditional $\mathrm{HI}$ and $\mathrm{VN}$ tests. H7N9 vaccines are apparently low immunogenic due to low $\mathrm{HI}$ and $\mathrm{VN}$ antibody titers they induced.

However, a latest study has demonstrated that evaluation of H7N9 vaccines based on only $\mathrm{HI}$ and $\mathrm{VN}$ may result in underestimation of immunogenicity of vaccines (Kamal et al., 2017). In their study, BALB/c mice were vaccinated with nonadjuvanted inactivated antigens derived from H7N7, H7N2, $\mathrm{H} 7 \mathrm{~N} 9, \mathrm{H} 1 \mathrm{~N} 1$, and $\mathrm{H} 3 \mathrm{~N} 2$ viruses. $\mathrm{H} 7$ subtype antigens induced significantly lower $\mathrm{HI}$ and $\mathrm{VN}$ titers compared to $\mathrm{H} 1$ and $\mathrm{H} 3$ subtypes, whereas they elicited comparable high IgG antibody titers measured by ELISA. This indicates that H7 subtype antigens are as immunogenic as the seasonal influenza antigens based on overall IgG antibody response.

Technically, both HI and VN antibodies are a part of IgG antibody, while the proportion of $\mathrm{HI}$ and $\mathrm{VN}$ antibodies induced by $\mathrm{H} 7$ antigens in total $\operatorname{IgG}$ antibody is significantly lower compared to their seasonal counterparts. A large fraction of antibody has no virus-neutralizing activity in vitro. More importantly, naïve mice passively immunized with the $\mathrm{H} 7$ immune serum were partially protected against lethal H7N7 challenge (Kamal et al., 2017). This suggests a significant role of non-neutralizing antibody (non-nAb) induced by $\mathrm{H} 7$ antigens in protective immunity in vivo. Similarly, two independent studies showed that both neutralizing and non-neutralizing antibody confer protection through divergent mechanisms (Henry Dunand et al., 2016; Tan et al., 2016). These findings indicate that the apparently low immunogenicity of H7N9 vaccines is at least partly related to measuring antibody titers with the traditional $\mathrm{HI}$ and VN assays, which may not provide a true measure of protective immunity associated with $\mathrm{H} 7$ immunization. The development of additional correlates of immunogenicity of $\mathrm{H} 7$ vaccines is needed.

\section{FACTORS AFFECTING ANTIBODY IMMUNITY INDUCED BY H7N9 VACCINE CANDIDATES}

H7N9 vaccines produced an equivalent level of overall $\operatorname{IgG}$ antibody with the seasonal influenza vaccines, whereas a large fraction of the antibody was not neutralizing in vitro. This is a remarkable signature of the quality of antibody immunity for H7N9 vaccines. Changes in the antigenic epitopes in HA may at least partially account for the production of non-nAb after $\mathrm{H} 7 \mathrm{~N} 9$ immunization.

In a recent report (Tan et al., 2016), neutralizing monoclonal antibody $(\mathrm{nAb})$ against the HA protein of a human H7N9 isolate bound to the epitope homologous to the antigenic site $\mathrm{A}$ of $\mathrm{H3}$ $\mathrm{HA}$, as determined by escape mutants assays. However, non-nAbs did not show any diminished binding to nAb-generated escape mutants, suggesting that non-nAbs did not bind to site A. In addition, since non-nAbs have no HI activity, it is likely that these antibodies would bind at some distance from the receptor binding site of HA. A panel of H7 HA mutants were screened for decreased binding of non-nAbs and diminished binding was observed for a mutant carrying R65K mutation. This site is located at the interface between the HA globular head and stalk region, which may be the target epitope recognized by non-nAbs.

Similarly, Dunand et al. cloned monoclonal nAbs and nonnAbs from plasmablasts isolated from $\mathrm{H} 7 \mathrm{~N} 9$-vaccinated humans (Henry Dunand et al., 2016). A competition assay showed that non-nAbs exhibited low levels of competition with stalk-reactive $\mathrm{nAbs}$, indicating that they bind to different epitopes. At low $\mathrm{pH}$, the HA stalk domain undergoes conformational changes to promote membrane fusion. nAbs binding to the stalk region can prevent membrane fusion by inhibiting conformational changes. An antibody binding test using viruses treated with various $\mathrm{pH}$-buffered (7.4-4.4) solutions revealed that exposure to low $\mathrm{pH}$ prevented binding of $\mathrm{mAbs}$ that recognize conformational epitopes on the pre-fusion HA. Interestingly, non-nAbs bound to $\mathrm{HA}$ in a post-fusion state ( $\mathrm{pH} 5.0$ and 4.4), suggesting that the epitope targeted was not affected by conformational changes and even become more accessible in the post-fusion state. Therefore, non-nAbs bind to epitopes outside of the classical binding sites, and these epitopes might be conformational epitopes. Although, epitopes targeted by non-nAb induced by H7N9 vaccines are still unidentified, it is clear that non-nAb plays a significant role in protection and the mechanism is the immune effects e.g. phagocytosis mediated by the interaction between IgG Fc and Fc receptors. 
Another factor influencing antibody immunity for H7N9 vaccines is the presence of Treg epitope in HA. An immunoinformatics team has identified that the $\mathrm{T}$ cell epitopes in $\mathrm{H} 7 \mathrm{HA}$ are highly conserved, on the $\mathrm{T}$ cell receptor face, with a large number of similar epitopes in human genome (Liu R. et al., 2015). This may lead to evasion of the HA protein from the immune response. Moreover, the magnitude of human $\mathrm{T}$ cell effector responses to individual H7N9 T cell peptides was inversely correlated with the peptide's resemblance to the host. An inhibitory T cell epitope in HA was identified, which suppressed responses to other $\mathrm{H} 7$ peptides and peptides of the seasonal influenza viruses in human peripheral blood mononuclear cells (PBMC). The underlying mechanism is that this peptide in HA has a high conservation with human genome, mediating "immune camouflage" and was shown to expand $\mathrm{CD}^{+}{ }^{+}$FoxP3 $^{+}$regulatory $\mathrm{T}$ cells (Treg), mediating a negative regulation of the immune response (Liu $\mathrm{R}$. et al., 2015). Thus, H7N9 AIVs may evade the immune response through "immune camouflage" and activation of Treg response. The presence of the inhibitory Tregitope in H7 HA was thought to be associated with low seroconversion rates and $\mathrm{HI}$ antibody titers induced by H7N9 vaccines in humans. However, there is no direct and solid evidence to support this speculation.

Since $\mathrm{HI}$ and VN titers are not sufficient to evaluate immunogenicity of H7N9 vaccines, it is necessary to investigate the effect of Treg in HA on serum IgG antibody. Wada et al. compared immunogenicity of $\mathrm{H} 7 \mathrm{~N} 9$ and $\mathrm{H} 3 \mathrm{~N} 2$ vaccines in a humanized mouse model (Wada et al., 2017). In BALB/c mice, an inactivated $\mathrm{H} 7 \mathrm{~N} 9$ vaccine and the recombinant $\mathrm{H} 7$ HA protein induced comparable HA-reactive IgG antibody titers as their $\mathrm{H} 3 \mathrm{~N} 2$ counterparts. However, in humanized mice that were transplanted with healthy human PBMC, IgG titers elicited by H7N9 antigens were significantly lower than that induced by $\mathrm{H} 3 \mathrm{~N} 2$ antigens. An explanation is that PBMC donors may have an $\mathrm{H} 3 \mathrm{~N} 2$ exposure history and H3N2 immunization activated the memory immunity. Intriguingly, when certain amino acids in the Treg epitope were mutated to those in $\mathrm{H} 3 \mathrm{~N} 2$, the optimized H7 antigens induced significantly higher IgG titers in humanized mice than did the wild-type HA. It is obvious that Treg exhibited a suppressive effect on IgG antibody titers measured by ELISA induced by H7N9 antigens in humanized mice. However, the optimized H7 HA failed to increase IgG antibody in mice transplanted with H3N2-primed murine splenocytes. It is probable that immune history of animal models may affect antibody immunity against influenza viruses and the function of Treg may be human leukocyte antigen (HLA)-restricted.

A further question we asked is that whether the localization of the $\mathrm{T}$ cell epitopes has some relationship with their immune regulatory effects. We found that 11 predicted $\mathrm{T}$ cell epitopes localize in different regions in H7 HA: 5 in the globular head, 4 in the stalk domain, 1 in the signal peptide and 1 in the TM domain (Table 2). It is noted that the inhibitory Treg epitope identified in the above immunoinformatics study localizes in the stalk region, indicating that the HA stalk may play a role in the regulation of the immune response. Intriguingly, the stalk region of H7N9 HA
TABLE 2 | Location of the predicted T cell epitopes in the HA protein.

\begin{tabular}{lccccc}
\hline Epitope & Position & \multicolumn{2}{c}{ Location in HA } & \\
\cline { 3 - 5 } & & Head & Stalk & SPc & TM $^{\mathbf{d}}$ \\
\hline CPRYVKQRSLLLATGMKNVPEa & $314-334$ & & \\
YAEMKWLLSNTDNAAFPQ & $155-172$ & \\
RIDFHWLMLNPNDTVTFS & $238-255$ \\
RASFLRGKSMGIQSG & $265-279$ \\
DKLYERVKRQLRENAEED & $455-472$ & & \\
AMGLVFICVKNGNMRCT & $541-557$ & & \\
GFTYSGIRTNGATSSCRR & $132-149$ & & \\
PGKFVNEEALRQILR & $107-121$ \\
MNTQILVFALIAIIPTNADKI & $1-21$ & \\
IDQITGKLNRLIEKT & $384-398$ & \\
NLPFQNIDSRAVGKC & $301-313$ &
\end{tabular}

a The inhibitory $T$ cell epitope identified using the immunoinformatics tools.

${ }^{b}$ The color values indicate the regions of $H A$.

${ }^{c} S P$, signal peptide.

dTM, transmembrane domain.

indeed had a suppressive effect on immunogenicity of the HA head (He et al., 2016). Different regions of HA, including the globular head, the stalk and the ectodomain (contains the head and stalk), fused with the intramolecular adjuvant IgG Fc were expressed. These vaccines were formulated with MF59 adjuvant for immunization study in mice. $\mathrm{HI}$ and $\mathrm{VN}$ antibody titers induced by the stalk-, head plus stalk-, and ectodomain-based vaccines were significantly lower than those induced by the HA head alone. This suggests that immunogenicity of HA head is significantly suppressed as long as the HA stalk is present, which provides important implications for rational design of HA-based vaccines. The author also surmised that domains or epitopes that are potentially suppressive to the neutralizing immunogenicity of HA head may exist in the stalk.

\section{PERSPECTIVES AND FUTURE DIRECTIONS}

Antibody response induced by H7N9 vaccines is qualitatively different from that induced by the seasonal influenza vaccines. H7N9 vaccines elicit low $\mathrm{HI}$ and VN titers but high overall IgG antibody titers. Thus the traditional $\mathrm{HI}$ and $\mathrm{VN}$ assays are not sufficient to evaluate immunogenicity of H7N9 vaccines. New immunological correlates, such as ELISA titers, to protection should be established. In addition, compared to the seasonal influenza vaccines, a large proportion of IgG antibody induced by $\mathrm{H} 7 \mathrm{~N} 9$ vaccines is non-nAb, indicating that epitopes recognized by non-nAb might be dominant epitopes in H7 HA. In addition, the immune history of animal models has an important impact on antibody immunity induced by influenza antigens, which should be taken into account when immunogenicity of H7N9 vaccines is evaluated. Although, there is no sufficient supporting evidence for the role of Treg, the presence of Treg epitope in H7 HA may partially account for low immunogenicity of H7N9 vaccines in a humanized mouse model and humans. 
These novel findings present critical implications for H7N9 vaccine design. There are several directions to improve or modify immunogenicity of H7N9 vaccines: (1) the molecular mechanism of the induction of non-nAbs by H7N9 antigens requires further studies; (2) the epitopes targeted by non$\mathrm{nAb}$ require to be identified and it may be doable to guide the host immune response toward the classical neutralizing epitopes by shielding non-nAb-recognized epitopes to enhance HI or VN antibody response; (3) the presence of Treg epitope in poultry and mammal models needs to be identified, and the mechanism of the inhibitory effect of Treg epitope on antibody response requires further understanding; (4) the inhibitory Treg epitope in HA may be related to low antibody levels in humans, it is reasonable to modify or remove this epitope or the stalk containing this epitope to enhance immunogenicity.

\section{REFERENCES}

Bahl, K., Senn, J. J., Yuzhakov, O., Bulychev, A., Brito, L. A., Hassett, K. J., et al. (2017). Preclinical and clinical demonstration of immunogenicity by mRNA vaccines against $\mathrm{H} 10 \mathrm{~N} 8$ and H7N9 influenza viruses. Mol. Ther. 25, 1316-1327. doi: 10.1016/j.ymthe.2017. 03.035

Bart, S. A., Hohenboken, M., Della Cioppa, G., Narasimhan, V., Dormitzer, P. R., and Kanesa-Thasan, N. (2014). A cell culturederived MF59-adjuvanted pandemic A/H7N9 vaccine is immunogenic in adults. Sci. Transl. Med. 6, 234ra255. doi: 10.1126/scitranslmed. 3008761

Blanchfield, K., Kamal, R. P., Tzeng, W. P., Music, N., Wilson, J. R., Stevens, J., et al. (2014). Recombinant influenza H7 hemagglutinins induce lower neutralizing antibody titers in mice than do seasonal hemagglutinins. Influenza Other Respir. Viruses 8, 628-635. doi: 10.1111/irv. 12285

Cao, W., Liepkalns, J. S., Hassan, A. O., Kamal, R. P., Hofstetter, A. R., Amoah, S., et al. (2016a). A highly immunogenic vaccine against A/H7N9 influenza virus. Vaccine 34, 744-749. doi: 10.1016/j.vaccine.2015. 12.062

Cao, W., Liepkalns, J. S., Kamal, R. P., Reber, A. J., Kim, J. H., Hofstetter, A. R., et al. (2016b). RIG-I ligand enhances the immunogenicity of recombinant H7HA protein. Cell. Immunol. 304-305, 55-58. doi: 10.1016/j.cellimm.2016. 04.004

Chen, Z., Baz, M., Lu, J., Paskel, M., Santos, C., Subbarao, K., et al. (2014). Development of a high-yield live attenuated H7N9 influenza virus vaccine that provides protection against homologous and heterologous $\mathrm{H} 7$ wild-type viruses in ferrets. J. Virol. 88, 7016-7023. doi: 10.1128/JVI.00100-14

Chia, M. Y., Hu, A. Y., Tseng, Y. F., Weng, T. C., Lai, C. C., Lin, J. Y., et al. (2015). Evaluation of MDCK cell-derived influenza H7N9 vaccine candidates in ferrets. PLOS ONE 10:e0120793. doi: 10.1371/journal.pone. 0120793

Chu, D. H., Sakoda, Y., Nishi, T., Hiono, T., Shichinohe, S., Okamatsu, M., et al. (2014). Potency of an inactivated influenza vaccine prepared from A/duck/Mongolia/119/2008 (H7N9) against the challenge with A/Anhui/1/2013 (H7N9). Vaccine 32, 3473-3479. doi: 10.1016/j.vaccine.2014. 04.060

Couch, R. B., Patel, S. M., Wade-Bowers, C. L., and Nino, D. (2012). A randomized clinical trial of an inactivated avian influenza A (H7N7) vaccine. PLoS ONE 7:e49704. doi: 10.1371/journal.pone. 0049704

Cox, M. M., Patriarca, P. A., and Treanor, J. (2008). FluBlok, a recombinant hemagglutinin influenza vaccine. Influenza Other Respir. Viruses 2, 211-219. doi: $10.1111 /$ j.1750-2659.2008.00053.x

\section{AUTHOR CONTRIBUTIONS}

$\mathrm{ZH}$ designed and drafted the manuscript. $\mathrm{XJ}$ and $\mathrm{XL}$ revised the manuscript.

\section{FUNDING}

This work was supported by the National Natural Science Foundation of China (31702243), the National Key Technologies R\&D Program of China (2015BAD12B01-3), by the National Key Research and Development Project of China (2016YFD0501601, 2017YFD0500701), by the Jiangsu Provincial Natural Science Foundation of China (BK20150444), by the earmarked fund for Modern Agro-industry Technology Research System (nycytx-41G07) and by A Project Funded by the Priority Academic Program Development of Jiangsu Higher Education Institutions (PAPD).

Cox, R. J., Madhun, A. S., Hauge, S., Sjursen, H., Major, D., Kuhne, M., et al. (2009). A phase I clinical trial of a PER.C6 cell grown influenza $\mathrm{H} 7$ virus vaccine. Vaccine 27, 1889-1897. doi: 10.1016/j.vaccine.2009.01.116

de Jonge, J., Isakova-Sivak, I., van Dijken, H., Spijkers, S., Mouthaan, J., de Jong, R., et al. (2016). H7N9 live attenuated influenza vaccine is highly immunogenic, prevents virus replication, and protects against severe bronchopneumonia in ferrets. Mol. Ther. 24, 991-1002. doi: 10.1038/mt. 2016.23

Duan, Y., Gu, H., Chen, R., Zhao, Z., Zhang, L., Xing, L., et al. (2014). Response of mice and ferrets to a monovalent influenza A (H7N9) split vaccine. PLoS ONE 9:e99322. doi: 10.1371/journal.pone.0099322

Fries, L. F., Smith, G. E., and Glenn, G. M. (2013). A recombinant viruslike particle influenza A (H7N9) vaccine. N. Engl. J. Med. 369, 2564-2566. doi: 10.1056/NEJMc1313186

Gao, R., Cao, B., Hu, Y., Feng, Z., Wang, D., Hu, W., et al. (2013). Human infection with a novel avian-origin influenza A (H7N9) virus. N. Engl. J. Med. 368, 1888-1897. doi: 10.1056/NEJMoa1304459

Griffin, M. R., Monto, A. S., Belongia, E. A., Treanor, J. J., Chen, Q., Chen, J., et al. (2011). Effectiveness of non-adjuvanted pandemic influenza A vaccines for preventing pandemic influenza acute respiratory illness visits in 4 U.S. communities. PLoS ONE 6:e23085. doi: 10.1371/journal.pone. 0023085

Grohskopf, L. A., Shay, D. K., Shimabukuro, T. T., Sokolow, L. Z., Keitel, W. A., Bresee, J., et al. (2013). Prevention and control of seasonal influenza with vaccines. Recommendations of the Advisory Committee on Immunization Practices-United States, 2013-2014. MMWR Recomm. Rep. 62, 1-43.

He, B., Xia, S., Yu, F., Fu, Y., Li, W., Wang, Q., et al. (2016). Putative suppressing effect of IgG Fc-conjugated haemagglutinin (HA) stalk of influenza virus H7N9 on the neutralizing immunogenicity of Fc-conjugated HA head: implication for rational design of HA-based influenza vaccines. J. Gen. Virol. 97, 327-333. doi: 10.1099/jgv.0.000365

Hekele, A., Bertholet, S., Archer, J., Gibson, D. G., Palladino, G., Brito, L. A., et al. (2013). Rapidly produced SAM((R)) vaccine against H7N9 influenza is immunogenic in mice. Emerg. Microbes Infect. 2, e52. doi: 10.1038/emi. 2013.54

Henry Dunand, C. J., Leon, P. E., Huang, M., Choi, A., Chromikova, V., Ho, I. Y., et al. (2016). Both neutralizing and non-neutralizing human H7N9 influenza vaccine-induced monoclonal antibodies confer protection. Cell Host Microbe 19, 800-813. doi: 10.1016/j.chom.2016.05.014

Isakova-Sivak, I., Korenkov, D., Smolonogina, T., Tretiak, T., Donina, S., Rekstin, A., et al. (2017). Comparative studies of infectivity, immunogenicity and cross-protective efficacy of live attenuated influenza vaccines containing nucleoprotein from cold-adapted or wild-type influenza virus in a mouse model. Virology 500, 209-217. doi: 10.1016/j.virol.2016. 10.027 
Iuliano, A. D., Jang, Y., Jones, J., Davis, C. T., Wentworth, D. E., Uyeki, T. M., et al. (2017). Increase in human infections with avian influenza A(H7N9) virus during the fifth epidemic - China, October 2016-February 2017. MMWR Morb. Mortal. Wkly. Rep. 66, 254-255. doi: 10.15585/mmwr.mm6609e2

Jackson, L. A., Campbell, J. D., Frey, S. E., Edwards, K. M., Keitel, W. A., Kotloff, K. L., et al. (2015). Effect of varying doses of a monovalent H7N9 influenza vaccine with and without AS03 and MF59 adjuvants on immune response: a randomized clinical trial. JAMA 314, 237-246. doi: 10.1001/jama. 2015.7916

Kamal, R. P., Blanchfield, K., Belser, J. A., Music, N., Tzeng, W. P., Holiday, C., et al. (2017). Inactivated H7 influenza virus vaccines protect mice despite low levels of neutralizing antibodies. J. Virol. doi: 10.1128/JVI.01202-17. [Epub ahead of print].

Kong, H., Zhang, Q., Gu, C., Shi, J., Deng, G., Ma, S., et al. (2015). A live attenuated vaccine prevents replication and transmission of $\mathrm{H} 7 \mathrm{~N} 9$ virus in mammals. Sci. Rep. 5:11233. doi: 10.1038/srep11233

Kreijtz, J. H., Wiersma, L. C., De Gruyter, H. L., Vogelzang-van Trierum, S. E., van Amerongen, G., Stittelaar, K. J., et al. (2015). A single immunization with modified vaccinia virus Ankara-based influenza virus $\mathrm{H} 7$ vaccine affords protection in the influenza $\mathrm{A}(\mathrm{H} 7 \mathrm{~N} 9)$ pneumonia ferret model. J. Infect. Dis. 211, 791-800. doi: 10.1093/infdis/jiu528

Li, Y., Qi, W., Qiao, J., Chen, C., Liao, M., and Xiao, C. (2017). Evolving HA and $\mathrm{PB} 2$ genes of influenza A (H7N9) viruses in the fifth wave - Increasing threat to both birds and humans? J. Infect. 75, 184-186. doi: 10.1016/j.jinf.2017.04.002

Liu, Q., Mena, I., Ma, J., Bawa, B., Krammer, F., Lyoo, Y. S., et al. (2015). Newcastle disease virus-vectored $\mathrm{H} 7$ and $\mathrm{H} 5$ live vaccines protect chickens from challenge with $\mathrm{H} 7 \mathrm{~N} 9$ or $\mathrm{H} 5 \mathrm{~N} 1$ avian influenza viruses. J. Virol. 89, 7401-7408. doi: 10.1128/JVI.00031-15 JVI.00031-15

Liu, R., Moise, L., Tassone, R., Gutierrez, A. H., Terry, F. E., Sangare, K., et al. (2015). H7N9 T-cell epitopes that mimic human sequences are less immunogenic and may induce Treg-mediated tolerance. Hum. Vaccin. Immunother. 11, 2241-2252. doi: 10.1080/21645515.2015.1052197

Liu, Y. V., Massare, M. J., Pearce, M. B., Sun, X., Belser, J. A., Maines, T. R., et al. (2015). Recombinant virus-like particles elicit protective immunity against avian influenza $\mathrm{A}(\mathrm{H} 7 \mathrm{~N} 9)$ virus infection in ferrets. Vaccine 33, 2152-2158. doi: 10.1016/j.vaccine.2015.03.009

Madan, A., Segall, N., Ferguson, M., Frenette, L., Kroll, R., Friel, D., et al. (2016). Immunogenicity and safety of an AS03-adjuvanted H7N9 pandemic influenza vaccine in a randomized trial in healthy adults. J. Infect. Dis. 214, 1717-1727. doi: 10.1093/infdis/jiw414

Mulligan, M. J., Bernstein, D. I., Winokur, P., Rupp, R., Anderson, E., Rouphael, N., et al. (2014). Serological responses to an avian influenza A/H7N9 vaccine mixed at the point-of-use with MF59 adjuvant: a randomized clinical trial. JAMA 312, 1409-1419. doi: 10.1001/jama.2014.12854

Nohynek, H., Jokinen, J., Partinen, M., Vaarala, O., Kirjavainen, T., Sundman, J., et al. (2012). AS03 adjuvanted AH1N1 vaccine associated with an abrupt increase in the incidence of childhood narcolepsy in Finland. PLOS ONE 7:e33536. doi: 10.1371/journal.pone.0033536

Ou, H., Yao, H., Yao, W., Wu, N., Wu, X., Han, C., et al. (2016). Analysis of the immunogenicity and bioactivities of a split influenza A/H7N9 vaccine mixed with MF59 adjuvant in BALB/c mice. Vaccine 34, 2362-2370. doi: 10.1016/j.vaccine.2016.03.037

Pan, W., Han, L., Dong, Z., Niu, X., Li, Z., Bao, L., et al. (2014). Induction of neutralizing antibodies to influenza A virus $\mathrm{H} 7 \mathrm{~N} 9$ by inactivated whole virus in mice and nonhuman primates. Antiviral Res. 107, 1-5. doi: 10.1016/j.antiviral.2014.04.003

Park, M. S., Steel, J., Garcia-Sastre, A., Swayne, D., and Palese, P. (2006). Engineered viral vaccine constructs with dual specificity: avian influenza and Newcastle disease. Proc. Natl. Acad. Sci. U.S.A. 103, 8203-8208. doi: $10.1073 /$ pnas. 0602566103

Partinen, M., Saarenpaa-Heikkila, O., Ilveskoski, I., Hublin, C., Linna, M., Olsen, P., et al. (2012). Increased incidence and clinical picture of childhood narcolepsy following the $2009 \mathrm{H} 1 \mathrm{~N} 1$ pandemic vaccination campaign in Finland. PLoS ONE 7:e33723. doi: 10.1371/journal.pone.0033723

Prabakaran, M., Kumar, S. R., Raj, K. V., Wu, X., He, F., Zhou, J., et al. (2014). Cross-protective efficacy of baculovirus displayed hemagglutinin against highly pathogenic influenza H7 subtypes. Antiviral Res. 109, 149-159. doi: 10.1016/j.antiviral.2014.06.017
Pushko, P., Pearce, M. B., Ahmad, A., Tretyakova, I., Smith, G., Belser, J. A., et al. (2011). Influenza virus-like particle can accommodate multiple subtypes of hemagglutinin and protect from multiple influenza types and subtypes. Vaccine 29, 5911-5918. doi: 10.1016/j.vaccine.2011.06.068

Pushko, P., Pujanauski, L. M., Sun, X., Pearce, M., Hidajat, R., Kort, T., et al. (2015). Recombinant $\mathrm{H} 7$ hemagglutinin forms subviral particles that protect mice and ferrets from challenge with H7N9 influenza virus. Vaccine 33, 4975-4982. doi: $10.1016 /$ j.vaccine.2015.07.026

Seo, S. H., and Kim, H. S. (2016). Inactivated antigen of the H7N9 influenza virus protects mice from its lethal infection. Viral Immunol. 29, 235-243. doi: $10.1089 /$ vim. 2015.0103

Smith, G. E., Flyer, D. C., Raghunandan, R., Liu, Y., Wei, Z., Wu, Y., et al. (2013). Development of influenza H7N9 virus like particle (VLP) vaccine: homologous A/Anhui/1/2013 (H7N9) protection and heterologous $\mathrm{A} /$ chicken/Jalisco/CPA1/2012 (H7N3) cross-protection in vaccinated mice challenged with H7N9 virus. Vaccine 31, 4305-4313. doi: 10.1016/j.vaccine.2013.07.043

Song, L., Xiong, D., Hu, M., Kang, X., Pan, Z., and Jiao, X. (2016). Immunopotentiation of different adjuvants on humoral and cellular immune responses induced by HA1-2 subunit vaccines of H7N9 influenza in mice. PLoS ONE 11:e0150678. doi: 10.1371/journal.pone.0150678

Song, L., Xiong, D., Kang, X., Yang, Y., Wang, J., Guo, Y., et al. (2015). An avian influenza A (H7N9) virus vaccine candidate based on the fusion protein of hemagglutinin globular head and Salmonella typhimurium flagellin. BMC Biotechnol. 15, 79. doi: 10.1186/s12896-015-0195-z

Tan, G. S., Leon, P. E., Albrecht, R. A., Margine, I., Hirsh, A., Bahl, J., et al. (2016). Broadly-reactive neutralizing and non-neutralizing antibodies directed against the $\mathrm{H} 7$ influenza virus hemagglutinin reveal divergent mechanisms of protection. PLoS Pathog. 12:e1005578. doi: 10.1371/journal.ppat.1005578

To, K. K., Zhang, A. J., Chan, A. S., Li, C., Cai, J. P., Lau, C. C., et al. (2015). Recombinant influenza A virus hemagglutinin HA2 subunit protects mice against influenza A(H7N9) virus infection. Arch. Virol. 160, 777-786. doi: 10.1007/s00705-014-2314-x

Wada, Y., Nithichanon, A., Nobusawa, E., Moise, L., Martin, W. D., Yamamoto, N., et al. (2017). A humanized mouse model identifies key amino acids for low immunogenicity of H7N9 vaccines. Sci. Rep. 7, 1283. doi: 10.1038/s41598-017-01372-5

Wang, X., Jiang, H., Wu, P., Uyeki, T. M., Feng, L., Lai, S., et al. (2017). Epidemiology of avian influenza A H7N9 virus in human beings across five epidemics in mainland China, 2013-17: an epidemiological study of laboratory-confirmed case series. Lancet Infect. Dis. 17, 822-832. doi: 10.1016/S1473-3099(17)30323-7

Wang, Y., Wu, J., Xue, C., Wu, Z., Lin, Y., Wei, Y., et al. (2017). A recombinant $\mathrm{H} 7 \mathrm{~N} 9$ influenza vaccine with the $\mathrm{H} 7$ hemagglutinin transmembrane domain replaced by the $\mathrm{H} 3$ domain induces increased cross-reactive antibodies and improved interclade protection in mice. Antiviral Res. 143, 97-105. doi: 10.1016/j.antiviral.2017.03.029

Wodal, W., Schwendinger, M. G., Savidis-Dacho, H., Crowe, B. A., Hohenadl, C., Fritz, R., et al. (2015). Immunogenicity and protective efficacy of a Vero cell culture-derived whole-virus H7N9 vaccine in mice and guinea pigs. PLoS ONE 10:e0113963. doi: 10.1371/journal.pone.0113963

Wong, S. S., Jeevan, T., Kercher, L., Yoon, S. W., Petkova, A. M., Crumpton, J. C., et al. (2014). A single dose of whole inactivated H7N9 influenza vaccine confers protection from severe disease but not infection in ferrets. Vaccine 32, 4571-4577. doi: 10.1016/j.vaccine.2014.06.016

Wongthida, P., Jengarn, J., Narkpuk, J., Koonyosying, P., Srisutthisamphan, K., Wanitchang, A., et al. (2016). In vitro and in vivo attenuation of Vesicular Stomatitis Virus (VSV) by phosphoprotein deletion. PLoS ONE 11:e0157287. doi: 10.1371/journal.pone.0157287

Wu, C. Y., Chang, C. Y., Ma, H. H., Wang, C. W., Chen, Y. T., Hsiao, P. W., et al. (2014). Squalene-adjuvanted H7N9 virus vaccine induces robust humoral immune response against $\mathrm{H} 7 \mathrm{~N} 9$ and H7N7 viruses. Vaccine 32, 4485-4494. doi: $10.1016 /$ j.vaccine.2014.06.043

Wu, U. I., Hsieh, S. M., Lee, W. S., Wang, N. C., Kung, H. C., Ou, T. Y., et al. (2017). Safety and immunogenicity of an inactivated cell culture-derived H7N9 influenza vaccine in healthy adults: a phase I/II, prospective, randomized, open-label trial. Vaccine 35, 4099-4104. doi: 10.1016/j.vaccine.2017. 06.044 
Yang, J. R., and Liu, M. T. (2017). Human infection caused by an avian influenza A (H7N9) virus with a polybasic cleavage site in Taiwan, (2017). J. Formos. Med. Assoc. 116, 210-212. doi: 10.1016/j.jfma.2017.02.011

Yang, X., Zhao, J., Wang, C., Duan, Y., Zhao, Z., Chen, R., et al. (2015). Immunization with a live attenuated H7N9 influenza vaccine protects mice against lethal challenge. PLOS ONE 10:e123659. doi: 10.1371/journal.pone.0123659

Zhang, F., Bi, Y., Wang, J., Wong, G., Shi, W., Hu, F., et al. (2017). Human infections with recently-emerging highly pathogenic H7N9 avian influenza virus in China. J. Infect. 75, 71-75. doi: 10.1016/j.jinf.2017.04.001
Conflict of Interest Statement: The authors declare that the research was conducted in the absence of any commercial or financial relationships that could be construed as a potential conflict of interest.

Copyright (c) $2017 \mathrm{Hu}$, Jiao and Liu. This is an open-access article distributed under the terms of the Creative Commons Attribution License (CC BY). The use, distribution or reproduction in other forums is permitted, provided the original author(s) or licensor are credited and that the original publication in this journal is cited, in accordance with accepted academic practice. No use, distribution or reproduction is permitted which does not comply with these terms. 